Tropical Journal of Pharmaceutical Research September 2014; 13 (9): 1401-1406

ISSN: $1596-5996$ (print); 1596-9827 (electronic)

(c) Pharmacotherapy Group, Faculty of Pharmacy, University of Benin, Benin City, 300001 Nigeria.

All rights reserved.

Available online at http://www.tjpr.org

Original Research Article

http://dx.doi.org/10.4314/tjpr.v13i9.4

\title{
In vivo Evaluation of a Cosmetic Emulsion Containing Soybean Extract for Anti-Aging
}

\author{
MK Waqas ${ }^{1}$, N Akhtar ${ }^{1}$, A Rasul ${ }^{2}$, SU Rashid ${ }^{3}$, R Mustafa ${ }^{1}$, BA Khan ${ }^{4}$ and G \\ Murtaza ${ }^{5 *}$ \\ ${ }^{1}$ Department of Pharmacy, Faculty of Pharmacy and Alternative Medicines, The Islamia University of Bahawalpur, Bahawalpur, \\ ${ }^{2}$ College of Pharmacy, Government College University Faisalabad, Faisalabad, ${ }^{3}$ Faculty of Pharmacy, University of Sargodha, \\ Sargodha, ${ }^{4}$ Faculty of Pharmacy, Gomal University, Dera Ismail Khan, ${ }^{5}$ Department of Pharmacy, COMSATS Institute of \\ Information Technology, Abbottabad, 22060 Pakistan
}

*For correspondence: Email: gmdogar356@gmail.com; Tel: 0092-314-2082826; Fax: 0092-922-383441

Received: 10 May 2014

Revised accepted: 6 August 2014

\begin{abstract}
Purpose: To develop and assess the anti-aging potential of a cosmetic W/O emulsion containing an extract of soybean, Glycine max (L.) Merr. Fabaceae.

Methods: This single-blind placebo-controlled study was performed in 11 healthy male human volunteers. A formulation comprising of $4 \%$ of concentrated extract of soybean was prepared by loading the extract in the internal aqueous phase of the emulsion. A control (base), consisting of the emulsion without the extract, was also prepared. Both formulations were applied to the cheeks of all volunteers for 12 weeks and their effect on different skin parameters, i.e., moisture contents, elasticity and surface evaluation of living skin (SELS) were assessed.

Results: The formulation containing $4 \%$ soybean extract showed significant $(p \leq 0.05)$ effects on skin elasticity and moisture contents but the base showed insignificant effect $(p>0.05)$. There was significant $(p \leq 0.05)$ decline in SELS, i.e., SEsc (skin scaliness, from $1.73 \pm 0.05$ to $1.66 \pm 0.06$ ), SEw (skin wrinkles, from $71.74 \pm 1.52$ to $68.51 \pm 1.64$ ), SEsm (skin smoothness, from $109.01 \pm 4.77$ to $102.03 \pm 4.23$ ), and SEr (skin roughness, from $4.04 \pm 0.09$ to $3.82 \pm 0.08$ ) parameters after application of the extract formulation for 12 weeks.

Conclusion: Topical application of the cosmetic emulsion containing soybean extract exerts potential skin anti-aging effects.
\end{abstract}

Keywords: Glycine max, Soybean, Anti-aging, Skin elasticity, Cosmetic emulsion, Surface evaluation of living skin (SELS), Skin moisture content

Tropical Journal of Pharmaceutical Research is indexed by Science Citation Index (SciSearch), Scopus, International Pharmaceutical Abstract, Chemical Abstracts, Embase, Index Copernicus, EBSCO, African Index Medicus, JournalSeek, Journal Citation Reports/Science Edition, Directory of Open Access Journals (DOAJ), African Journal Online, Bioline International, Open-J-Gate and Pharmacy Abstracts

\section{INTRODUCTION}

Skin aging is a complicated procedure promoted by persistent exposure to UV radiation. UV radiations produce the ROS (reactive oxygen species) assisting in collagen deficit and ultimately skin wrinkling. Skin aging is depicted by increased wrinkling, decline in elasticity, uneven pigmentation, increased roughness and dryness [1]. Oil-in-water or water-in-oil emulsions are among the most frequently used colloidal systems, largely present in many fields including cosmetics, pharmaceuticals, food, paints, and petrochemicals $[2,3]$. A wide variety of cosmetic emulsions are used as bases for skincare products for healthy and diseased skin [4]. 
Botanical extracts that support the health, texture, and integrity of the skin, hair, and nails are widely used in cosmetic formulations. They form the largest category of cosmeceutical additives found in the marketplace today due to the rising consumer interest and demand for natural products [5]. Soybean seeds extract has positive research support for its antioxidant, antiproliferative, and anticarcinogenic activities. This extract is used to treat hyperhidrosis in Asian medicine [6]. Topical application of soy has been used to reduce hyperpigmentation, enhance skin elasticity, control oil production, moisturize the skin, and delay hair regrowth. Soy also has the potential to decrease photoaging of the skin and prevent skin cancers through the antioxidant effects of its metabolites [7]. The major components of soy are phospholipids (45 $60 \%$ ), such as phosphatidyl choline, and essential fatty oils $(30-35 \%)$. The minor components include the most active compounds, such as isoflavones, saponins, essential amino acids, phytosterols, calcium, potassium, iron, and the proteases soybean trypsin inhibitor and Bowman-Birk inhibitor.

The purpose of the present work is to develop a stable formulation of $\mathrm{W} / \mathrm{O}$ following inclusion of botanical extract containing soybean seeds extract and its effects were analyzed on various parameters related to skin aging.

\section{EXPERIMENTAL}

\section{Materials}

Cetyl dimethicone copolyol (ABIL EM 90) was purchased from the Franken Chemicals, Germany, n-hexane and paraffin oil were purchased from Merck KGaA Darmstadt Germany and ethanol (95\%) was purchased from the BDH England. Distilled water was prepared in the cosmetics laboratory of the Department of Pharmacy, The Islamia University of Bahawalpur, Pakistan. Soy bean seeds were obtained in May, 2012 from a Hamid Pensaar, Bahawalpur, Pakistan and authenticated by Prof. Mukhtar Ahmad, Cholistan Institute of Desert Plants Studies (CIDS), The Islamia University of Bahawalpur, Pakistan. For future reference, a voucher specimen collected and identified by the CIDS, and specimen was deposited by the Herbarium, The Islamia University of Bahawalpur (voucher no. GM-SD-7-16-27). Rotary evaporator (Eyela, Japan) was used for solvent evaporation. Mexameter MPA 5 (Courage + Khazaka, Germany) used for the determination of skin melanin and skin erythema.

\section{Preparation of extract}

Plant material ( $1 \mathrm{~g}$ per sample) was ground to a fine powder in a mill and extracted for $20 \mathrm{~min}$ with ethanol: hexane 1:1 $(50 \mathrm{ml})$ solvent mixture under sonication in an ultrasonic bath at ambient temperature. The extract residue was removed by filtration through layers of muslin cloth. The extract was concentrated at rotary evaporator. Concentrated extract was filtered through Whatman No. 1 filter paper and was stored extract in refrigerator.

\section{Evaluation of antioxidant activity of plant extract}

1,1-Diphenyl-2-picrylhydrazyl (DPPH) was prepared by taking $1 \mathrm{mg}$ of DPPH in $25 \mathrm{ml}$ methanol to make $100 \mu \mathrm{l}$ solution. The stable DPPH radical was used for the determination of antioxidant activity. Different concentrations of extract in respective solvents were added at an equal volume of $10 \mu \mathrm{l}$ to $90 \mu \mathrm{l}$ of $100 \mu \mathrm{M}$ methanolic DPPH solution in a total volume of $100 \mu \mathrm{l}$ in 96 well plates. The contents were mixed and incubated at $37{ }^{\circ} \mathrm{C}$ for $30 \mathrm{~min}$. Ascorbic acid was used as standard antioxidant. The reduction in absorbance was measured at $517 \mathrm{~nm}$ by using microplate reader; in comparison with the control solution (maximum absorption). The decrease in absorbance indicates increased scavenging activity. The DPPH radical scavenging activity is mentioned as percentage [7].

\section{Preparation of cosmetic emulsions}

Soybean seeds ( $1 \mathrm{~g}$ per sample) were ground to a fine powder in a mill and extracted for $48 \mathrm{~h}$ with ethanol: hexane 1:1 $(50 \mathrm{ml})$ solvent mixture under sonication in an ultrasonic bath at ambient temperature. The extract residue was removed by filtration through layers of muslin cloth. The extract was concentrated at rotary evaporator. Concentrated extract was filtered through Whatman No. 1 filter paper and was stored in refrigerator. For the formulation oily phase composed of paraffin oil (16\%) and emulsifier Abil-EM90 (3\%) were heated together up to $75 \pm$ $1{ }^{\circ} \mathrm{C}$. At the same time distilled water (quantity sufficient to make $100 \%$ ) was heated at the same temperature and then $4 \%$ soy bean extract was added in it. Next, aqueous phase was added to the oil phase drop by drop. Stirring was continued at $2000 \mathrm{rpm}$ by the mechanical mixer for about 15 min until complete aqueous phase was added, 2 to 3 drops of rose oil were added during this stirring time to give good fragrance to the formulation. 
Thereafter, the speed of the mixer was reduced to $1000 \mathrm{rpm}$ for homogenization, for a period of 5 min, and then the speed of the mixer was further reduced to $500 \mathrm{rpm}$ for $5 \mathrm{~min}$ for complete homogenization; until the emulsion cooled to room temperature. The base was also prepared by the same method and with same ingredients but without the soy bean seeds extract. Both base and the test formulation were found stable after evaluating $\mathrm{pH}$, centrifugation, electrical conductivity, phase separation, organoleptic and physical characteristics (color, creaming and liquefaction) and the temperature stability tests at storage conditions of $8 \pm 0.5,25 \pm 0.5,40 \pm 0.5$, and also at $40 \pm 0.5{ }^{\circ} \mathrm{C} / 70 \% \mathrm{RH}$ (relative humidity) for a period of 3 months.

\section{Ethical approval}

This work was authorized by the Board of Advanced Studies and Research (BASR), and its Ethical Committee for in vivo Studies (reference no. 4710/Acad.), The Islamia University of Bahawalpur, Pakistan and was directed according to the Principles of Good Clinical Practice [8] and the International Guidelines of Helsinki Declaration [9].

\section{Patch test}

To determine the skin irritation assessment, on the first day of study Patch tests were performed on forearms of each human volunteer. An area of $5 \mathrm{~cm} \times 4 \mathrm{~cm}$ was marked on both the forearms of all the volunteers Basic values for erythema and melanin were measured with the help of Mexameter. $1.0 \mathrm{~g}$ of base and formulation each were applied to the $5 \mathrm{~cm} \times 4 \mathrm{~cm}$ marked regions separately on each forearm. Surgical dressing was used to cover the marked area of right and left forearm. After 48 hours application of emulsion, it was removed and the forearms were washed with physiological saline and were observed for any skin redness/irritation by a dermatologist. The measurements of erythema and melanin were repeated on both forearms.

\section{Study protocol}

Eleven volunteers were selected whose ages were in between 25 and 35 years. Male volunteers were included in this work and consent forms were taken. Prior to the tests, a dermatologist for any serious skin disease or damage especially on cheeks and forearms examined the volunteers. Each volunteer was provided with the two emulsions. One emulsion was the base $(B)$ with no extract and other was the active formulation $(F)$ containing soybean extract. The study was designed single blinded placebo-controlled for the comparisons of two emulsions. The investigations were achieved on cheeks of volunteers. They were also given instructions regarding way of application. The volunteers were advised to use an amount which can be absorbed easily on the skin. Each volunteer applied emulsion for the period of 12 weeks. Values for different parameters were taken in controlled room temperature $25 \pm 1{ }^{\circ} \mathrm{C}$ and $45 \pm 2 \%$ relative humidity. The site of measurement was the whole cheek. Every volunteer was instructed to come for measurement on $2^{\text {nd }}, 4^{\text {th }}, 6^{\text {th }}, 8^{\text {th }}, 10^{\text {th }}$ and $12^{\text {th }}$ week. Values for each parameter was measured three times $(n=3)$ and average of the values has been noted.

\section{Anti-aging study}

The evaluation of skin microrelief parameters was done by using Visio Scan®) VC98 (Courage and Khazaka, Germany). The skin elasticity was measured by an Elastometer (Skin Elastometer®EM 25, Courage and Khazaka, Germany). A skin capacitance meter (Corneometer® MPA 5, Courage and Khazaka, Germany) was used for the determination of skin moisture contents.

\section{Data analysis}

The evaluation of data was done by using SPSS version 17.0 according to two-way ANOVA for assessing variation between different time intervals defining $5 \%$ level of significance and Paired samples t-test for determining variation between the two preparations. Standard error of means (SEM) was calculated for every mean value.

Percent change in individual values for each parameter, taken weekly, were computed as in Eq 1.

$\%$ Change $=\{(A-B) / B\}^{*} 100$

where $A=$ individual value of any parameter on 2nd, 4th, 6th, 8th, 10th and 12th week, and B = the value of the parameter at $0 \mathrm{~h}$, i.e., at commencement of the test.

\section{RESULTS}

For eleven volunteers, changes in skin moisture contents are shown in Figure 1. In this work base enhances the moisture contents of skin to some degree but there was consistent increase in skin moisture contents when formulation was applied throughout the study duration. Insignificant effects $(p>0.05)$ were observed by the base while the formulation presented significant $(p \leq$ 

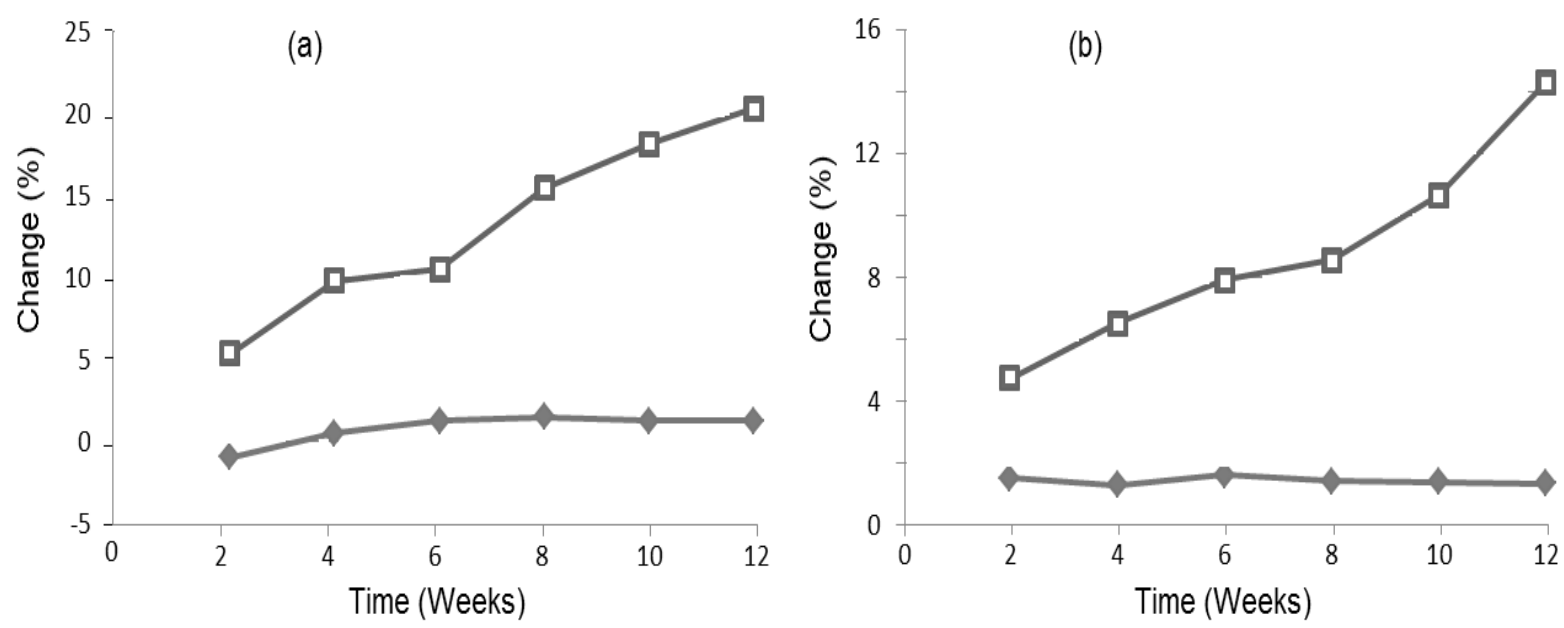

Figure 1: Change in (a) skin moisture and (b) elastic content after application of base ( $\bullet$ ) and soybean extract formulation (口)

Table 1: SELS values (mean \pm SD) of formulations

\begin{tabular}{lccccc}
\hline Parameter & Emulsion & $\mathbf{0 ~ h}$ & $\mathbf{1}$ month & $\mathbf{2}$ months & 3 months \\
\hline Ser & Base & $4.05 \pm 0.09$ & $4.04 \pm 0.07$ & $4.04 \pm 0.09$ & $4.04 \pm 0.09$ \\
& Formulation & $4.04 \pm 0.09$ & $4.00 \pm 0.08$ & $3.95 \pm 0.08$ & $3.82 \pm 0.08$ \\
SEsc & Base & $1.74 \pm 0.05$ & $1.74 \pm 0.05$ & $1.73 \pm 0.05$ & $1.73 \pm 0.05$ \\
& Formulation & $1.73 \pm 0.05$ & $1.70 \pm 0.05$ & $1.68 \pm 0.05$ & $1.66 \pm 0.06$ \\
SEsm & Base & $108.99 \pm 4.91$ & $109.98 \pm 4.91$ & $108.64 \pm 4.89$ & $107.82 \pm 4.92$ \\
& Formulation & $109.01 \pm 4.77$ & $108.72 \pm 4.81$ & $106.72 \pm 4.66$ & $102.03 \pm 4.23$ \\
Sew & Base & $71.84 \pm 1.54$ & $72.83 \pm 1.55$ & $71.84 \pm 1.54$ & $70.93 \pm 2.00$ \\
& Formulation & $71.74 \pm 1.52$ & $71.93 \pm 1.53$ & $69.96 \pm 1.65$ & $68.51 \pm 1.64$ \\
\hline
\end{tabular}

Note: SEr = skin roughness; Sew = skin wrinkles; SEsm = skin smoothness; SEsc = skin scaliness

0.05) effects on skin moisture contents regarding with time. Throughout the study duration significant differences in moisture values were evaluated after application of formulation. The irregular pattern in the values of skin elasticity was observed by the base but there was regular increase in skin elasticity after the application of formulation throughout the study duration shown in Figure 2. Test formulation showed significant $(p<0.05)$ while the base had insignificant $(p>$ $0.05)$ effects on skin elasticity. Significant $(p<$ 0.05 ) differences in skin elasticity values were noticed after application of the formulation throughout the study duration. Data about SELS parameters SEw, SEsc, SEsm and SEr before the application of emulsions and then at 1st, 2nd and 3rd months of the study duration are given in Table 1. Insignificant $(p>0.05)$ effect was produced by the base on the SEr parameter of skin and the statistically insignificant $(p>0.05)$ effect was produced by formulation at all reading intervals. Significant $(p>0.05)$ effects were observed for formulation when paired sample ttest was performed. It was observed that active formulation showed a consistent decrease in values of smoothness, scaliness, roughness and wrinkles.

\section{DISCUSSION}

Moisturizing remedy involves renovating the skin barrier, restoring the water contents, ability to retain and redistribute water and sustaining the integrity and skin complexion [10]. Significant differences in moisture values were evaluated after application of formulation throughout the study duration. Enhancement of skin moisture contents after the application of formulation may because of soy bean extract which contain flavonoids. Soybean flavonoids enhance the skin moisture contents due to swelling of corneocytes on skin surface [11]. Consequently, it may be used in moisturizing cosmetic formulations and also as a complement in the treatment of dry skin.

The elasticity of the skin is mainly due to the collagen fibers which is a main constituent of the dermis of the skin. It constitutes about $70 \%$ of the total dry weight of the skin. Vitamin $\mathrm{C}$ stimulates the collagen production in the skin fibroblasts and its deficiency will result in harmful 
effects on the skin [12]. The collagen is vulnerable to the UV radiations of the sunlight which causes structural changes in them resulting in photo aging. Photo aging is due to the oxidative effects of these radiations on the collagen of the skin [13]. The improvement in skin elasticity after application of formulation is due to the presence phytoestrogens [14]. Soybean extracts was found to instigate the synthesis of collagen and elastin, and to boost the correct assembly of new elastin fibers, providing a complete protection and restoration to the dermal extracellular matrix [15]. Soybeans extract also protected elastic fibers produced by cultured fibroblasts from degradation by exogenously-added elastases [16].

Skin surface is illustrated by several parameters like smoothness (SEsm), wrinkling (SEw), roughness (SEr) and scaliness (SEsc). Skin surface is assessed both qualitatively and quantitatively by the use of SELS parameters [17]. The proportion of dark pixels is calculated by roughness parameter SEr.A higher SEr value reflects more roughness while a lower value $\mathrm{SEr}$ represents more smoothness. SEsm is the smoothness parameter which measures the mean depth and width of wrinkles. SEsc shows the level of dryness of the skin. Stratum corneum dehydration is evaluated by SEsc. The smaller value of SEsc indicates more hydration level with less scaliness [18]. The width and number of skin wrinkles are assessed by SEw which is evaluated by proportion of vertical and horizontal wrinkles. A higher value of SEw proves more and wider wrinkles [17]. It was observed that active formulation showed a consistent decrease in values of smoothness, scaliness, roughness and wrinkles.

The decrease in the average values of skin smoothness in contrast to skin roughness represents that formulation acquires the antiaging effects. Lower value of SEsc designates that there was high skin moisture content. This indicates that formulation has the capability to increase the skin moisture. The smaller values of SEw shown by the extract formulation represent a reduction in fine wrinkles due to the enhancement of skin complexion by stimulation of elastin and collagen synthesis and promotion of production of crosslinked, stable elastin fibers by soybean extract. Augmentation of skin surface parameters can be ascribed to the presence of a rich content of antioxidant flavonoids, known as genistein and daidzein, in soybeans. These substances have been classified as phytoestrogens, since they are plant derivatives with chemical structure similar to human estrogen. Topical estrogens have been shown to function as antiaging cosmeceuticals by increasing skin thickness, promoting the collagen synthesis and prevention of skin from oxidative damage and photoaging [18].

\section{CONCLUSION}

The findings indicate that topical application of cosmetic water-in-oil emulsion incorporating $4 \%$ soybean extract exerts potential antiaging effects. Furthermore, the formulation has no detrimental effects and thus can be used safely. It is also a suitable alternative to most commercial formulations that utilize synthetic bases. The non-invasive technique used in this study is a suitable approach for the evaluation of antiaging properties of skin topical agents.

\section{REFERENCES}

1. Gilchrest BA. Skin aging and photoaging: an overview. J Am Acad Dermatol 1989; 21: 610-613.

2. Lin $\mathrm{CH}$, Sheu JY, Wu HL, Huang YL. Determination of hydroquinone in cosmetic emulsion using microdialysis sampling coupled with highperformance liquid chromatography. J Pharm Biomed Anal 2005; 38: 414-419.

3. Saikia AP, Ryakala VK, Sharma $P$, Goswami $P$, Bora $U$. Ethnobotany of medicinal plants used by Assamese people for various skin ailments and cosmetics. J Ethnopharmacol 2006; 106: 149-157.

4. Hall WL, Vafeiadou K, Hallund J, Bügel S, Koebnick $C$, Reimann M, Ferrari M, Branca F, Talbot D, Dadd T. Soy-isoflavone-enriched foods and inflammatory biomarkers of cardiovascular disease risk in postmenopausal women: interactions with genotype and equol production. Am J Clin Nutr 2005; 82: 1260-1268.

5. Cassidy A, Albertazzi $P$, Nielsen IL, Hall W, Williamson G, Tetens I, Atkins S, Cross $H$, Manios $Y$, Wolk A. Critical review of health effects of soybean phytooestrogens in post-menopausal women. P Nutr Soc 2006; 65: 76-92.

6. Akiyama $T$, Ishida $J$, Nakagawa $S$, Ogawara $H$, Watanabe $S-i$, Itoh $N$, Shibuya $M$, Fukami $Y$. Genistein, a specific inhibitor of tyrosine-specific protein kinases. J Biol Chem 1987; 262: 5592-5595.

7. Waqas MK, Shah PA, Akhtar N, Murtaza G. Screening of Various Botanical Extracts for Antioxidant Activity Using DPPH Free Radical Method. Afr J Tradit Complement Altern Med 2013; 10: 452-455.

8. The European Agency for the Evaluation of Medicinal Products, Human Medicines Evaluation Unit, International Conference on Harmonisation: Guideline for Good Clinical Practice [EMEAWeb site], http://www.emea.eu.int.

9. World Medical Association Declaration of Helsinki: Ethical principles of medical research involving human

Trop J Pharm Res, September 2014; 13(9): 1405 
subjects. http://www.wma.net/en/30publications/10 plicies/b3/index.html.

10. Grahame $R$, Holt $P$. The influence of ageing on the in vivo elasticity of human skin. Gerontol 1969; 15: 121 . 139.

11. Farage $M$, Miller $K$, Elsner $P$, Maibach $H$. Intrinsic and extrinsic factors in skin ageing: a review. Int $\mathrm{J}$ Cosmetic Sci 2008; 30: 87-95.

12. Quella SK, Loprinzi CL, Barton DL, Knost JA, Sloan JA, LaVasseur BI, Swan D, Krupp KR, Miller KD, Novotny $P J$. Evaluation of soy phytoestrogens for the treatment of hot flashes in breast cancer survivors: $A$ North Central Cancer Treatment Group Trial. J Clin Oncol 2000; 18: 1068-1068.

13. Draelos ZD. Topical and oral estrogens revisited for antiaging purposes. Fertil Steril. 2005; 84: 291-292.

14. Zhao R, Bruning E, Rossetti D, Starcher B, Seiberg M, lotsova-Stone $V$. Extracts from Glycine max (soybean) induce elastin synthesis and inhibit elastase activity. Exp dermatol 2009; 18: 883-886.

15. Heinrich U, Tronnier H, Stahl W, Bejot M, Maurette JM. Antioxidant supplements improve parameters related to skin structure in humans. Skin Pharmacol Phys 2006; 19: 224-231.

16. Primavera G, Berardesca E. Clinical and instrumental evaluation of a food supplement in improving skin hydration. Int J Cosmetic Sci 2005; 27: 199-204.

17. Udompataikul M, Sripiroj $P$, Palungwachira $P$. An oral nutraceutical containing antioxidants, minerals and glycosaminoglycans improves skin roughness and fine wrinkles. Int J Cosmetic Sci 2009; 31: 427-435.

18. Kim SY, Kim SJ, Lee JY, Kim WG, Park WS, Sim YC, Lee SJ. Protective effects of dietary soy isoflavones against UV-induced skin-aging in hairless mouse model. J Am Coll Nutr 2004; 23: 157-162. 